\title{
Sleep disorders in children
}

\author{
Timothy F. Hoban
}

The Michael S. Aldrich Sleep Disorders Center, Departments of Pediatrics and Neurology, University of Michigan, Ann Arbor, Michigan, USA

Address for correspondence: Timothy F. Hoban, MD, L3227 Women's Hospital, 1500 East Medical Center Drive, SPC 5203 , Ann Arbor, Michigan 48109-5203. Voice: 734-936-4179; fax: 734-763-7551. thoban@umich.edu

\begin{abstract}
Although sleep disorders such as insomnia and obstructive sleep apnea are common in both children and adults, the clinical features and treatments for these conditions differ considerably between these two populations. Whereas an adult with obstructive sleep apnea typically presents with a history of obesity, snoring, and prominent daytime somnolence, a child with the condition is more likely to present with normal body weight, tonsillar hypertrophy, and inattentiveness during school classes. The adult with suspected sleep apnea almost always undergoes a baseline polysomnogram and proceeds to treatment only if this test confirms the diagnosis, while many children with suspected sleep apnea are treated empirically with adenotonsillectomy without ever receiving a sleep study to verify the diagnosis. This article reviews sleep disorders in children, with a particular focus on age-related changes in sleep, conditions that primarily affect children, and disorders for which clinical manifestations and treatment differ substantially from the adult population.
\end{abstract}

Keywords: pediatric sleep disorders; insomnia; circadian rhythm disorders; night waking; parasomnias; obstructive sleep apnea; restless legs syndrome; periodic limb movement disorder; rhythmic movement disorder

\section{Evolution of normal sleep during infancy and early childhood}

Healthy term infants spend in excess of $15 \mathrm{~h}$ asleep each day in the form of brief $2-4$ h sleep periods. Periods of sleep and wakefulness are initially distributed equally across daytime and nighttime hours with little day-to-day consistency. Longer periods of wakefulness develop by 6 weeks of age, and by the age of 3 months, these periods are predictably distributed during the daytime or early evening hours. ${ }^{1}$ As circadian regulatory mechanisms become stronger and entrained by recurring environmental and social cues, sleep lengthens and consolidates during nighttime hours in a process known as settling. Although $70 \%$ of infants settle by 3 months of age, $10 \%$ do not achieve sustained nighttime sleep during the first year of life. ${ }^{2}$

The toddler and preschool years are characterized by additional declines in daily sleep duration from an average of $13.9 \mathrm{~h}$ at 1 year of age to $11.4 \mathrm{~h}$ at age $5{ }^{3}$ Daytime napping begins to subside during the second year of life, with most children giving up regular naps around age 3 .
The quantitative changes of sleep during early childhood are accompaniment by significant qualitative changes in sleep architecture, which are demonstrable on electroencephalography (EEG) and polysomnography (PSG). Rapid eye movement (REM) sleep constitutes $50 \%$ of total sleep time during early infancy, declining by age 5 to $20 \%$, the proportion exhibited by older children and adults. ${ }^{4}$ Ultradian sleep cycles of REM and nonREM sleep states are substantially shorter for infants (50-60 $\mathrm{min})$ than for adults (90-100 $\mathrm{min})$.

\section{Night waking and insomnia in younger children}

It is important to recognize that some degree of nighttime waking is normal in young children, particularly during early infancy, when circadian mechanisms are still immature and nighttime feedings a necessity for most infants until about 6 months of age. ${ }^{5}$ Among a cohort of children aged 11 to 27 months with no reported sleep problems, actigraphic monitoring of nighttime sleep revealed an average of two awakenings per night. ${ }^{6}$ Night 
waking is considered problematic when it is excessively prolonged or frequent for age, or when it is excessively disruptive to other members of the household. Overall, about $20 \%$ of children below age 2 years exhibit night waking considered concerning by parents. ${ }^{7}$

A variety of predisposing influences may be associated with problematic night waking, some of which relate to expected developmental changes during early childhood. Nighttime feeding represents a biological need for younger infants until 6 months of age, which may persist as a learned behavior beyond that time. Near 1 year of age, separation anxiety may disrupt previously well-established sleep schedules and result in significant distress during episodes of night waking. Cosleeping with a parent is also associated with increased risk for arousal and awakening during nighttime sleep. ${ }^{8}$

Insomnia in younger children usually takes the form of bedtime resistance, in which a child may cry, repeatedly attempt to leave the room, or engage in other behaviors intended to forestall sleep onset. These behaviors often result in considerable distress and frustration for both child and parent. Although bedtime struggles are at least occasionally observed in most younger children, frequent or sustained bedtime resistance is exhibited by $20 \%$ of children between 1 and 3 years of age, and by $10 \%$ of 4 -year-olds. ${ }^{9}$

Several factors exhibit strong influences upon settling problems at bedtime. Children whose temper- amental characteristics are not conducive to selfsettling are at increased risk for bedtime struggles. Children who lack structured bedtime routines or have irregular sleep habits represent another highrisk population. Other extrinsic influences that may impede settling at bedtime include anxiety, acute illness, medication side effects, and coexisting medical conditions such as attention deficit hyperactivity disorder, cerebral palsy, autism, and other developmental disabilities. ${ }^{10}$

Many young children exhibiting habitual bedtime struggles or night waking for at least 3 months duration meet diagnostic criteria for behavioral insomnia of childhood (Table 1). ${ }^{11}$ The essential feature of this condition is that the difficulty falling asleep or staying asleep is related to an identified behavioral etiology-either inappropriate sleep onset associations or inadequate limit setting. In the sleep-onset association type, a young child habitually settles to sleep under circumstances such as being rocked, watching television, or having a parent present. Affected children become so reliant upon these habitual sleep associations that they are unable to fall asleep in their absence. Absence of the desired circumstances then results in emotional distress, which can disrupt sleep onset both at bedtime and following the physiological awakenings that punctuate normal childhood sleep.

The limit-setting type of behavioral insomnia of childhood occurs when parental or caregiver response to bedtime resistance or night waking is

\section{Table 1. Diagnostic criteria for behavioral insomnia of childhood}

1. Symptoms must meet criteria for insomnia based on parent or caregiver report

2. The child's symptoms are consistent with either the sleep-onset association or limit-setting types of insomnia defined below:

a. Sleep-onset association type includes each of the following:

i. Falling asleep is a protracted process requiring special conditions.

ii. Sleep-onset associations are highly demanding or problematic.

iii. In the absence of the typical associated conditions, sleep onset is substantially delayed or disrupted.

iv. Nighttime awakenings require intervention by the parent or caregiver for the child to return to sleep.

b. Limit-setting type includes each of the following:

i. The child has difficulty initiating or maintaining sleep.

ii. The child stalls or refuses to go to bed at an appropriate time or refuses to return to bed following nighttime waking.

iii. The parent or caregiver demonstrates inappropriate or insufficient limit setting relative to the child's bedtime or sleep behavior.

3. The sleep disturbance is not better explained by another sleep, medical, neurological, or behavioral disorder.

Adapted from the International Classification of Sleep Disorders, second edition. ${ }^{11}$ 
either suboptimal or inconsistent. Whereas firm, consistent limit setting by parents in response to bedtime struggles ultimately reduces the duration and severity of the episodes, inappropriate or inconsistent caregiver response to the child's behavior tends to perpetuate or worsen the problem.

\section{Treatment of sleeplessness during early childhood}

Treatment for milder forms of sleeplessness in infants and young children begins with establishing good "sleep hygiene." Use of a structured, ageappropriate bedtime routine helps younger children settle into a quiet but relaxed state conducive to sleep onset. Routines for younger children may include bathing, changing, tooth brushing, reading stories, and being "tucked in." Potentially stimulating activities such as watching television and vigorous play should be avoided during the lead-in to bedtime.

Establishing a regular sleep schedule and an appropriate sleep environment are additional important elements of sleep hygiene for younger children. Maintaining regular bedtime and waking times 7 days per week may strengthen and entrain circadian mechanisms to promote rapid sleep onset near the desired bedtime. Eliminating daytime sleep except for age-appropriate naps may also augment sleepiness at bedtime. The optimal sleep environment for most young children is one that is quiet and kept at a stable, comfortable temperature. Although dark sleep environments are most conducive to sleep, use of dim night lights is appropriate for young children who are fearful of the dark.

Treatments for behavioral insomnia of and other persistent forms of sleeplessness during early childhood consist primarily of structured behavioral interventions. ${ }^{12}$ Interventions considered to be well established for use in younger children include:

Extinction (systematic ignoring). This technique involves placing the child in bed and then ignoring any agitation or inappropriate behaviors that the child exhibits until morning with the exception of legitimate concerns regarding safety or illness.

Graduated extinction. This technique, described by Douglas and Richman ${ }^{13}$ and popularized by Ferber, ${ }^{14}$ specifies schedules by which parents ignore tantrums or inappropriate behavior for gradually increasing intervals before briefly checking on and reassuring the child.
Positive routines/faded bedtime with response cost. This technique couples a quiet but enjoyable bedtime routine with fading of bedtime toward the child's time of habitual sleep onset. Response cost involves taking the child briefly out of bed if not falling asleep, an application of stimulus control.

Parent education and anticipatory guidance. This technique, usually coupled with other specific behavioral interventions, educates parents and caregivers about early childhood sleep and the treatable influences that may promote or disrupt it.

A systematic review ${ }^{15}$ and practice parameters ${ }^{16}$ regarding behavioral treatments of sleep problems in infants and young children reported that several varieties of extinction-based therapies, positive routines with bedtime fading, and parent education were all individually effective therapies but found that there was insufficient evidence to recommend one therapy over another.

Drug treatment of sleeplessness in infants and young children has received remarkably little scientific study despite reportedly frequent use of such agents in this age group. ${ }^{17}$ Controlled trials of trimeprazine tartrate, a sedating antihistamine, for treatment of night waking in young children have yielded conflicting results. ${ }^{18,19}$ Although melatonin, diphenhydramine, and chloral hydrate are been used for treatment of sleep problems in younger children, reports of efficacy are largely anecdotal apart from a single placebo-controlled trial of diphenhydramine in infants which did not identify significant short-term benefits for treatment of night waking. ${ }^{20,21}$

\section{Evolution of normal sleep during later childhood and adolescence}

The sleep of school-age and adolescent children evolves at a more modest pace than that of younger children. Average daily sleep duration declines from $11 \mathrm{~h}$ at 6 years of age to $10 \mathrm{~h}$ at age $9,9 \mathrm{~h}$ at age 13 , and $8 \mathrm{~h}$ at age $16 .{ }^{3}$ Maturation of sleep architecture is characterized by gradual reductions in the proportion of deep non-REM sleep and compensatory increases in the proportion of light non-REM sleep stages.

Normal sleep in preadolescent children is accompanied by high degrees of baseline alertness during daytime as measured by the multiple sleep latency test (MSLT). Mean sleep latency for healthy children 
on the MSLT averages $19 \pm 1.6 \mathrm{~min}$, considerably higher than the 10-13 min range considered normal for adults. ${ }^{22}$ Given this high level of baseline alertness, prominent sleepiness in a preadolescent child is seldom encountered except in the context of a severe underlying sleep disorder or significant sleep deprivation.

During adolescence, daytime sleepiness as measured by the MSLT increases, which may reflect the effects of average sleep duration being insufficient relative to overall sleep needs. ${ }^{23}$ In addition, many healthy adolescents demonstrate tendencies toward delayed circadian phase, trending toward progressively later bedtimes and-particularly on nonschool days - waking times.

\section{Insomnia in older children and adolescents}

Insomnia, daytime sleepiness, and other subjectively reported sleep problems remain common for school-aged children and adolescents, affecting 10$30 \%$ of elementary schoolchildren and a similar proportion of adolescents. ${ }^{24-26}$ Social, environmental, and developmental influences have significant impact on sleep for this age group. For example, older children and adolescents gain increasing autonomy regarding their bedtime and sleep schedule compared with younger children, often resulting in irregular sleep habits and chronically insufficient sleep. The widespread availability of televisions, music players, and computers in the bedrooms of school-age children and the frequent use of these devices during nighttime hours is associated with increased risk for a variety of sleep disturbances. ${ }^{27,28}$ Use of mobile telephones after bedtime is prevalent among secondary schoolchildren and associated with increased risk for daytime sleepiness. ${ }^{29}$

Another strong influence on the sleep patterns of older school-age children is the well-recognized tendency toward delayed circadian sleep phase that develops near adolescence. ${ }^{26}$ Circadian rhythm disorder, delayed sleep phase type, formerly called delayed sleep phase syndrome, may be diagnosed when this tendency results in chronic or recurrent inability of the child to fall asleep and wake up at conventional and socially acceptable times. ${ }^{11}$ When this condition results in chronically insufficient sleep, it is often accompanied by daytime napping, which may make it more difficult for the child to fall asleep at the desired bedtime. Habitually later bedtime and waking time on nonschool nights-common in adolescentstend to perpetuate or worsen the tendency toward delayed sleep phase. ${ }^{30}$ Delayed sleep phase can be mimicked or exacerbated when medication or caffeine use result in insomnia with chronic sleep deprivation or when homework or other late-evening activities habitually delay sleep onset.

Psychophysiological insomnia in older children is characterized by chronic preoccupation with and excessive anxiety regarding sleep, which results in heightened arousal that delays sleep onset. ${ }^{11}$ Affected children often report anxiety or identifiable rumination in conjunction with insomnia when trying to fall asleep at bedtime but are sometimes able to fall asleep easily during sedentary activities or when away from home.

\section{Treatment of insomnia in older children and adolescents}

Treatment of insomnia during later childhood is seldom effective unless all pertinent contributory influences are identified and addressed in the treatment plan. For adolescents in particular, it is common to identify multiple concurrent influences such as irregular sleep schedule, excessive caffeine use, insufficient time allotted for sleep, and tendency toward delayed sleep phase.

Establishing or optimizing appropriate "sleep hygiene" is a necessary foundation for other aspects of treatment. Optimal bedtime and waking time should be identified based on the child's age, school schedule, and individual sleep needs. Because regularity of sleep schedule helps maintain circadian entrainment, delayed bedtimes and "sleeping in" on nonschool days should be avoided whenever possible. Potentially stimulating activities such as vigorous exercise, video gaming, and consumption of caffeinated beverages should be discouraged during the evening hours. Eliminating use of electronic devices such as televisions, music players, and portable telephones in the bedroom near bedtime helps promote optimal sleep onset associations and a dark, quiet environment more conducive to sleep initiation and maintenance.

Insomnia related primarily to delayed circadian sleep phase can be treated in several ways. Mildly delayed sleep phase often responds to consistent enforcement of the desired sleep schedule by 
maintaining target bedtime and waking times 7 days weekly while eliminating any daytime napping. An alternative and comparably effective strategy consists of gradually advancing the child's bedtime and waking time earlier by 10-15 min each day until the target schedule is achieved.

More severe or resistant forms of delayed sleep phase often respond to chronotherapy, a process by which bedtime and waking time are delayed by $2-3 \mathrm{~h}$ nightly until the desired sleep schedule is attained. ${ }^{31}$ Although this treatment is safe, highly effective, and does not require use of medication, the effects of treatment are typically maintained only if the target sleep schedule is maintained rigorously 7 days weekly following completion of the treatment protocol. Light therapy, a treatment designed to influence circadian rhythmicity via structured daily exposure to high-intensity light, has also been reported to be effective in treating delayed sleep phase in children and adolescents. ${ }^{32,33}$

Drug treatment for insomnia in older children and adolescents has received extremely limited scientific study despite reports that $10-12 \%$ of adolescents use medication for treatment of sleep problems. ${ }^{17,34,35}$ Melatonin has been reported to be effective for treatment of insomnia in school-aged children and adolescents in both uncontrolled ${ }^{36,37}$ and placebo-controlled ${ }^{38}$ studies. Although reports of melatonin treatment for insomnia in children have assessed doses ranging from 0.3 to $20 \mathrm{mg},{ }^{39}$ the side effects, long-term safety, and optimal dosing of this agent for pediatric use have not been well established.

Numerous other medications are used off label for the treatment of insomnia in older children in spite of a paucity of scientific data assessing overall safety and efficacy. These include antihistamines, benzodiazepines, tricyclic agents, alpha-adrenergic agonists, antipsychotics, herbal preparations, and newer short-acting hypnotics. ${ }^{17}$ Pharmacology and dosing for these agents relative to pediatric sleep disorders were recently reviewed by Pelayo and Dubik. $^{21}$

\section{Non-rapid eyemovement arousal parasomnias: night terrors and sleepwalking}

Night terrors, sleepwalking, and confusional arousals in children are thought to represent forms of incomplete arousal from deep non-REM sleep. These aberrant arousals are associated with a spectrum of behaviors that may range from quiet somnambulism to extreme agitation. Episodes occur most commonly during the early hours of nighttime sleep, when deep non-REM sleep stages are predominantly distributed, particularly in younger children. Children are difficult to awaken during the event, and if awakened, demonstrate little or no recall for the episode.

Exacerbations of non-REM arousal parasomnias are often triggered by sleep deprivation or intercurrent illness. Reports of human leukocyte antigen associations and familial clustering for patients with arousal parasomnias suggest that these disorders are subject to strong genetic influences. ${ }^{40,41}$ Limited evidence suggests that obstructive sleep apnea or restless legs syndrome may represent a treatable precipitant of these parasomnias for some children. ${ }^{42}$

Night terrors and confusional arousals affect up to $17 \%$ of children ${ }^{43}$ but are most commonly observed in toddlers and preschoolers. The semiology of events ranges from moaning or quiet crying with the child remaining in bed to extreme agitation accompanied by attempts to flee the room. Episodes may last as long as $1 \mathrm{hr}$, but most typically remit within minutes. Affected children usually have little or no subsequent recollection of events.

Although $40 \%$ of children exhibit at least one episode of sleepwalking, frequent sleepwalking affects only $2-3 \%$, with peak prevalence near 10 years of age ${ }^{43,44}$ Episodes consist of quiet ambulation lasting several minutes, which may include semipurposeful activities such wandering to the parent's room or urinating in inappropriate locations. Sleepwalking children seldom sustain serious injury in their familiar home environment, but risk for injury increases when children leave the house during an episode or are sleeping in an unfamiliar environment.

Treatment of the non-REM arousal parasomnias in children should commence with the identification and treatment of precipitants such as sleep deprivation or obstructive sleep apnea. It is important to safeguard the child's sleep environment and limit the child's access to potentially hazardous areas inside and outside the house through judicious use of locks or alarms. Use of scheduled awakenings is often effective for children whose sleepwalking or night terrors occur at predictable times of 
the night. ${ }^{45,46}$ Drug treatment using clonazepam may effective for children having particularly frequent or severe forms of sleepwalking and night terrors. $^{47,48}$

\section{Other childhood parasomnias: nightmares and enuresis}

Nightmares arise from REM sleep and are thought to result from awakening during a frightening dream. Agitation following awakening is sometimes considerable, but the fact that the child awakens fully, responds to consolation by the parent, and has recollection of dream content helps distinguish nightmares from night terrors. Nightmares usually arise during the latter half of the child's nighttime sleep period, when REM periods are usually longest and most prevalent. Nightmares during childhood are common, affecting over half of early school-aged children on an occasional basis but only $3 \%$ of children more than once weekly. ${ }^{49}$

Occasional nightmares in otherwise healthy children represent a self-limited problem that seldom requires further assessment or treatment. Persistent nightmares which are unexpectedly frequent, prolonged, or violent in content, however, may signal a need for further investigation of potential medical and psychological causes.

Sleep enuresis in children is characterized by recurrent involuntary voiding during sleep at a level that is inappropriate for age, defined by the International Classification of Sleep Disorders as more than twice weekly for children older than 5 years of age. ${ }^{11}$ Sleep enuresis is defined as primary if the child has never remained consistently dry during sleep and secondary if the child had previously been consistently dry for at least 6 months. Nocturnal enuresis becomes less common with advancing age, affecting $15 \%$ of 3 - to 10 -year-olds, but only $2 \%$ of 13 -year-olds. ${ }^{43}$ Boys are affected three times as frequently as girls. Postulated causes of primary enuresis include immaturity of arousal mechanisms, reduced functional bladder capacity, and genetic predisposition.

Treatment of primary sleep enuresis in children varies depending on age and severity. Most younger children with primary enuresis outgrow the condition by adolescence without treatment. Children with frequent or persistent forms of primary enuresis often respond to behaviorally based therapies and conditioning programs that incorporate fluid restriction, planned awakenings, bladder training exercises, behavioral incentives, and enuresis alarms either independently or in combination..$^{50,51}$ Drug treatment using desmopressin ${ }^{52}$ and imipramine ${ }^{53}$ is also effective for many children with sleep enuresis, but reports of hyponatremia ${ }^{54}$ and other potentially serious mediation-related side effects ${ }^{53}$ suggest that these agents are best used within the context of a comprehensive and closely supervised treatment program.

Children with secondary sleep enuresis or refractory primary enuresis sometimes require evaluation for underlying urological or sleep disorders. Sleep enuresis is frequently associated with symptoms of obstructive sleep apnea in children. ${ }^{55,56}$ In one series of 144 children with PSG-confirmed obstructive sleep apnea, 42 (29\%) demonstrated sleep enuresis prior to treatment. ${ }^{57}$ Among the 27 children with enuresis treated with adenotonsillectomy, 11 (41\%) experienced resolution of bedwetting within 1 month following surgery.

\section{Sleep-related movement disorders: restless legs syndrome and rhythmic movement disorder}

Restless legs syndrome (RLS) and periodic limb movement disorder (PLMD) are distinct but related conditions only recently recognized in the pediatric age group. Symptoms of RLS in children include dysesthesias or urges to move the legs, which are usually most prominent at night and relieved by movement. ${ }^{11}$ Children with growing pains often meet diagnostic criteria for RLS as well. ${ }^{58}$ Childhood PLMD is characterized by excessive periodic limb movements occurring over five times per hour of sleep during PSG, accompanied by clinical complaints of disturbed sleep. ${ }^{11}$ Although RLS and PLMD are often comorbid conditions in both children and adults, the association is not obligate and either condition may present independently.

Leg restlessness and growing pains affect up to $17 \%$ and $8 \%$ of children, respectively, ${ }^{59}$ but only $2 \%$ of children meet diagnostic criteria for clinically definite RLS. ${ }^{60}$ Multiple studies have reported association between childhood RLS/PLMD and symptoms of attention deficit hyperactivity disorder (ADHD); ${ }^{61-63}$ however, the strength and clinical significance of this association remains incompletely defined. Several reports suggest that genetic 
Table 2. Diagnostic criteria for pediatric obstructive sleep apnea

1. Parent or caregiver report of snoring or other obstructive symptoms during sleep

2. Parent or caregiver report of at least one associated symptom:

a. Paradoxical chest wall motion during inspiration

b. Movement arousals

c. Excessive perspiration

d. Unusual sleeping positions, for example, neck hyperextension

e. Daytime symptoms: hyperactivity, aggressive behavior, or sleepiness

f. Impaired growth

g. Headache upon waking

h. Secondary enuresis

3. PSG documents at least one scoreable respiratory event per hour of sleep (events lasting at least two respiratory cycles in duration)

4. PSG demonstrates either a or b:

a. At least one of the following findings:

i. Frequent arousals associated with increased respiratory effort

ii. Desaturation with apneic events

iii. Hypercapnia in sleep

iv. Excessively negative esophageal pressure fluctuations

b. Periods of abnormal gas exchange during sleep (hypoxemia, hypercapnea, or both) with snoring, paradoxical chest wall motion, and at least one of the following:

i. Frequent arousals during sleep

ii. Excessively negative esophageal pressures swings

5. The disorder is not better explained by other sleep disorders, medical or neurological conditions, or by medication or substance use

Adapted from the International Classification of Sleep Disorders, second edition. ${ }^{11}$

influences ${ }^{64}$ and iron deficiency states ${ }^{65}$ may play a causative role in childhood RLS. Treatment options for childhood RLS/PLMD consist of iron supplementation for children with low ferritin levels or other evidence of iron deficiency, ${ }^{66,67}$ or judicious use of dopaminergic agonists. ${ }^{68,69}$

Sleep-related rhythmic movement disorder (RMD) is characterized by recurrent and wellstereotyped rhythmic behaviors associated with sleep. ${ }^{11,70}$ Common behaviors include head banging (jactatio capitis nocturna), head or body rolling, and body rocking. Movements typically involve the large muscle groups of the head, trunk, or limbs at a frequency of 0.5 to $2 \mathrm{~Hz}$, often accompanied by synchronous humming or moaning vocalizations. Episodes of rhythmic movement typically last for several minutes but may recur throughout the night. RMD is unique among the parasomnias insofar as episodes may commence during wakefulness but persist during and following transition into sustained sleep.
Sleep-related rhythmic behaviors are observed in a majority of infants, but these behaviors subside before 5 years of age in most children. ${ }^{71}$ By early adolescence, only $3 \%$ of children manifest prominent rhythmic behaviors associated with sleep. ${ }^{43}$ For most affected children, RMD is a self-limited condition that does not require treatment. For children exhibiting particularly prolonged or violent forms of RMD, treatment options include benzodiazepines, behavioral therapies, or use of padding and protective helmets. $^{72}$

\section{Sleep-disordered breathing in children}

The first modern description of obstructive sleep apnea (OSA) in children was provided by Guilleminault and colleagues in $1976 .{ }^{73}$ This and subsequent reports have characterized childhood OSA as a condition characterized by upper airway obstruction that disturbs sleep quality, sometimes associated with recurrent oxyhemoglobin desaturation or (less commonly) hypercapnia. 
Table 3. Clinical features of obstructive sleep apnea in children compared to adults

\begin{tabular}{|c|c|c|}
\hline & Children & Adults \\
\hline \multicolumn{3}{|l|}{ Physical characteristics } \\
\hline Gender & $\begin{array}{l}\text { Younger children: sexes equally affected } \\
\text { Adolescents: males > females }\end{array}$ & Primarily males, postmenopausal females \\
\hline Peak age & $2-8$ years & Middle age and older \\
\hline Body weight & Usually normal, occasionally overweight & Most often obese \\
\hline Upper airway & $\begin{array}{l}\text { Adenotonsillar enlargement frequent } \\
\text { Redundant soft tissue occasional }\end{array}$ & $\begin{array}{l}\text { Adenotonsillar enlargement occasional } \\
\text { Redundant soft tissue frequent }\end{array}$ \\
\hline \multicolumn{3}{|c|}{ Symptoms during sleep } \\
\hline Snoring & Frequent, continuous or intermittent & Frequent, often interrupted by pauses \\
\hline Witnessed apnea & Occasional & Frequent \\
\hline \multicolumn{3}{|l|}{ PSG characteristics } \\
\hline Obstruction & Prolonged partial obstruction $>$ intermittent & Cyclical intermittent obstruction \\
\hline Sleep architecture & Normal $>$ fragmented & Frequent arousals with sleep fragmentation \\
\hline \multicolumn{3}{|l|}{ Secondary symptoms } \\
\hline Daytime sleepiness & Most often absent or intermittent & Frequent, usually prominent \\
\hline Neurobehavioral & Inattention, hyperkinesis, disturbed behavior & Cognitive slowing, increased accident risk \\
\hline Cardiovascular & Hypertension, cor pulmonale & Hypertension, heart disease, stroke \\
\hline
\end{tabular}

Criteria for the diagnosis of childhood OSA, summarized in Table 2, were established by the International Classification of Sleep Disorders, second edition. ${ }^{11}$ This classification, however, did not specify criteria for other varieties of sleep-disordered breathing sometimes observed in children. Children with neuromuscular disorders, underlying lung disease, or severe varieties of partial airway obstruction sometimes exhibit sleep-related hypoventilation, characterized by persistent hypercapnia during sleep. ${ }^{74}$ Upper airway resistance syndrome (UARS), a condition in which prolonged partial airway obstruction during sleep disturbs sleep quality in the absence of apnea or desaturation, has also been described in children. ${ }^{75}$

It is estimated that about $2 \%$ of children suffer from OSA. ${ }^{76}$ The condition may develop at any time during childhood but is most frequent between 2 and 8 years of age, the age range when adenotonsillar size is largest relative to size of the airway. OSA is equally frequent in boys and girls until adolescence, when the condition becomes more common in males. Other genetic, neurological, and craniofacial conditions are associated with increased risk for the development of childhood OSA, particularly
Down syndrome, in which half of children may be affected. ${ }^{77,78}$

The clinical features of OSA in children are distinct from those of adults, as summarized in Table 3. Snoring is usually present in affected children, but frequency and severity are highly variable. Other common symptoms during nighttime sleep include mouth breathing, restlessness, diaphoresis, and unusual sleeping positions. Symptoms upon waking may include sore throat, dry mouth, headache, and transient grogginess. Daytime somnolence is seldom prominent in younger children with OSA unless obstruction is particularly severe.

A number of studies suggest that disturbances of attention, behavior, and academic performance are commonly associated with childhood OSA and may improve following treatment. Sleep-disordered breathing was identified in 18\% of 297 first-grade children with poor academic achievement in one study. ${ }^{79}$ Upon reassessment 1 year later, the 24 affected children who had been treated with adenotonsillectomy demonstrated significant academic improvement $(P<0.001)$ compared with untreated and unaffected children. In a more recent study of 78 children undergoing adenotonsillectomy for 
suspected sleep-disordered breathing, 22 (28\%) met $D S M-I V$ criteria for the diagnosis of ADHD preoperatively, but only half of these children still met criteria for the diagnosis of ADHD 1 year following surgery. ${ }^{80}$

The long-term effects and clinical sequelae of childhood OSA remain incompletely understood. Multiple reports suggest associations between childhood OSA and hypertension. ${ }^{81,82}$ Reversible cor pulmonale has also been reported in severely affected children. ${ }^{83,84}$

The physical examination of children with OSA is often normal, but findings such as tonsillar hypertrophy, narrow upper palate, and low-lying soft palate are frequently identified. Mouth breathing with elongated facial appearance is sometimes apparent in children with underlying adenoidal hypertrophy. Other occasionally encountered physical features associated with increased risk for sleeprelated airway obstruction include maxillary or mandibular hypoplasia, macroglossia, or nasal septal deviation. Although body weight is normal for most children with OSA, obesity nevertheless represents a clearly identifiable risk factor, particularly in adolescents. ${ }^{85,86}$

The diagnosis of childhood OSA (Table 2) is established on the basis of both clinical and polysomnographic criteria. ${ }^{87}$ Lab-based PSG is now widely but not universally available for children, but the number of sleep laboratories having substantial experience in pediatric studies remains limited. Specific norms for the interpretation of PSG in children have been established, ${ }^{74,88}$ which are distinct from adult norms and reflect the fact that adult criteria often fail to identify children with serious obstruction. ${ }^{89}$ PSG can be customized through addition of end-tidal carbon dioxide or esophageal pressure monitoring if sleeprelated hypoventilation or upper airway resistance syndrome are clinically suspected. A variety of techniques are available for making PSG more "child friendly." 90

Adenotonsillectomy (AT) is the most common treatment of childhood OSA. This procedure has been used for decades to alleviate upper airway obstruction in children with suspected or documented OSA. ${ }^{91}$ Although previously issued practice guidelines ${ }^{92}$ and meta-analysis of published research ${ }^{93}$ had estimated that the procedure was effective in curing childhood OSA in over $75 \%$ of cases, these estimates were limited by substantial variability in the criteria used to define childhood OSA and measure outcome. Several large, recent studies using contemporary criteria and PSG techniques to study the efficacy of AT in treating childhood OSA reported that complete normalization of the PSG may occur in only $25 \%{ }^{94}$ to $50 \%{ }^{95}$ of treated children. These and other data suggest that healthcare practitioners should remain vigilant for residual or recurrent ${ }^{96,97}$ OSA in children treated with AT.

Nasal CPAP (continuous positive airway pressure) is an alternative first-line treatment for OSA in children of all ages. ${ }^{98}$ Advantages of CPAP include the facts that the treatment is nonsurgical, generally safe, and effective for treatment of sleepdisordered breathing related to factors independent from adentonsillar hypertrophy. Limitations of this treatment include the fact that some childrenespecially those who are young or have concurrent developmental disabilities-may not acclimate easily to use of the device, although specific desensitization techniques are often helpful in these patients. ${ }^{99}$

Alternative and investigational medical treatments for childhood OSA include use of supplemental oxygen and positional therapy. Use of nasal steroid preparations may be effective in some cases; ${ }^{100,101}$ however, the long-term efficacy and safety of this treatment in children have not been established. Surgical treatments sometimes used for treatment of children with complicated OSA include uvulopaltopharyngoplasty (UPPP), distraction osteogenesis, and mandibular or maxillary advancement procedures. Tracheostomy is used only as a last resort in children whose OSA is both severe and refractory to more conventional therapies.

\section{Childhood hypersomnias}

Excessive daytime sleepiness in children is most often secondary to other sleep problems such as insufficient sleep or more severe forms of obstructive sleep apnea. Nevertheless, primary hypersomnias such as narcolepsy and Kleine-Levin syndrome are occasionally observed in children.

As is the case for adults, narcolepsy in children is characterized by excessive daytime somnolence, which may be accompanied by cataplexy, sleep paralysis, and hallucinations at sleep onset or offset. Onset of symptoms most commonly occurs 
during the second decade, ${ }^{102}$ usually in the form of isolated sleepiness, which can develop in either an abrupt or insidiously progressive fashion. Cataplexy, sleep paralysis, and other associated symptoms of narcolepsy, when present, may develop months to years after the child becomes somnolent. Evolution of severe somnolence or cataplexy at an especially young age or in an atypical fashion is sometimes observed in children with other medical conditions such as Niemann-Pick disease type $\mathrm{C}^{103}$ or PraderWilli syndrome. ${ }^{104}$

The diagnosis of narcolepsy in children is based on the presence of typical clinical features, supplemented by nocturnal PSG - to screen for other sleep disorders that may cause excessive sleepiness - and daytime MSLT interpreted using pediatric norms. ${ }^{22}$ Most children with narcolepsy demonstrated mean sleep latency of fewer than $7 \mathrm{~min}$ and multiple sleeponset REM periods on their MSLT studies. ${ }^{105}$

Treatment of narcolepsy in children parallels that of adults in most respects. Although detailed practice parameters ${ }^{106}$ and guidelines for treatment of narcolepsy ${ }^{107}$ have been published, these do not specifically address drug treatment in children, which is undertaken on an off-label basis. Daytime sleepiness in narcoleptic children is treated using modafinil, stimulants, and other wake-promoting medications, sometimes supplemented by planned daytime naps. Cataplexy often responds to administration of tricyclic antidepressant agents or selective serotonin reuptake inhibitors. Sodium oxybate has been found to be effective in the treatment of sleepiness and cataplexy in adults with narcolepsy, and limited data suggest that this agent may be effective for affected children as well. ${ }^{108}$

Kleine-Levin syndrome, also known as recurrent hypersomnia, is a rare disorder characterized by recurrent episodes of excessive sleepiness, often accompanied by concurrent behavioral disturbances, which may include irritability, aggression, marked changes in appetite, and hypersexual behavior. ${ }^{11}$ Episodes last from several days to several weeks and occur between once and 10 times yearly. KleineLevin syndrome most commonly affects adolescent males, and limited evidence suggests that the severity and duration of the condition may be worse for patients who are older at the age of onset. ${ }^{109}$

The pathophysiology of Kleine-Levin syndrome remains poorly understood, but SPECT studies suggest that intermittent disturbances of hypothalamic, thalamic, and fronto-temporal function may coincide with episodes. ${ }^{10,111}$ Treatment of the condition is symptomatic and often includes mood stabilizers and wake-promoting medications such as modafinil.

\section{Conflicts of interest}

The author declares no conflicts of interest.

\section{References}

1. Coons, S., C. Guilleminault, S. Coons \& C. Guilleminault. 1982. Development of sleep-wake patterns and non-rapid eye movement sleep stages during the first six months of life in normal infants. Pediatrics 69: 793 798.

2. Guilleminault, C. \& T.F. Anders. 1976. The pathophysiology of sleep disorders in pediatrics. Part II. Sleep disorders in children. Adv. Pediatrics 22: 151-174.

3. Iglowstein, I., O.G. Jenni, L. Molinari \& R.H. Largo. 2003. Sleep duration from infancy to adolescence: reference values and generational trends [see comment]. Pediatrics 111: 302-307.

4. Anders, T.F. \& C. Guilleminault. 1976. The pathophysiology of sleep disorders in pediatrics. Part I. Sleep in infancy. Adv. Pediatrics 22: 137-150.

5. Ferber, R.. 1996. Childhood sleep disorders. Neurol. Clin. 14: 493-511.

6. Sadeh, A., P. Lavie, A. Scher, et al. 1991. Actigraphic home monitoring of sleep-disturbed and control infants and young children: a new method for pediatric assessment of sleep-wake patterns. Pediatrics 87: 494499.

7. Sheldon, S.H. 2005. Disorders of initiating and maintaining sleep. In Principles and Practice of Pediatric Sleep Medicine. Sheldon, S.H., R. Ferber \& M.H. Kryger, Eds.: 127-160. Philadelphia: Saunders.

8. Mosko, S., C. Richard \& J. McKenna. 1997. Infant arousals during mother-infant bed sharing: implications for infant sleep and sudden infant death syndrome research. Pediatrics 100: 841-849.

9. Ramchandani, P., L. Wiggs, V. Webb \& G. Stores. 2000. A systematic review of treatments for settling problems and night waking in young children. BMJ 320: 209-213.

10. Stores, G. 1999. Children's sleep disorders: modern approaches, developmental effects, and children at special risk. Dev. Med. Child Neurol. 41: 568-573.

11. Anonymous. 2005. The International Classification of Sleep Disorders, 2nd edn. American Academy of Sleep Medicine. Westchester. 
12. Kuhn, B.R. \& A.J. Elliott. 2003. Treatment efficacy in behavioral pediatric sleep medicine. J. Psychosom. Res. 54: 587-597.

13. Douglas, J \& N. Richman. 1984. My Child Won't Sleep. Penguin Books. Harmondsworth.

14. Ferber, R. 1985. Solve Your Child's Sleep Problems. Simon \& Schuster. New York.

15. Mindell, J.A., B. Kuhn, D.S. Lewin, et al. 2006. Behavioral treatment of bedtime problems and night wakings in infants and young children [erratum appears in Sleep. 2006 Nov 1;29(11):1380]. Sleep 29: 1263-1276.

16. Morgenthaler, T.I., J. Owens, C. Alessi, et al. 2006. Practice parameters for behavioral treatment of bedtime problems and night wakings in infants and young children. Sleep 29: 1277-1281.

17. Owens, J.A., C.L. Rosen \& J.A. Mindell. 2003. Medication use in the treatment of pediatric insomnia: results of a survey of community-based pediatricians. Pediatrics 111(5 Pt 1): e628-e635.

18. France, K.G., N.M. Blampied \& P. Wilkinson. 1999. A multiple-baseline, double-blind evaluation of the effects of trimeprazine tartrate on infant sleep disturbance. Exp. Clin. Psychopharmacol. 7: 502-513.

19. Simonoff, E.A. \& G. Stores. 1987. Controlled trial of trimeprazine tartrate for night waking. Arch. Dis. Child. 62: 253-257.

20. Merenstein, D., M. Diener-West, A.C. Halbower, et al. 2006. The trial of infant response to diphenhydramine: the TIRED study-a randomized, controlled, patientoriented trial. Arch. Pediatr. Adolesc. Med. 160: 707712.

21. Pelayo, R. \& M. Dubik. 2008. Pediatric sleep pharmacology. Semin. Pediatr. Neurol. 15: 79-90.

22. Hoban, T.F. \& R.D. Chervin. 2001. Assessment of sleepiness in children. Semin. Pediatr. Neurol. 8: 216-228.

23. Carskadon, M.A. \& W. Dement. 1987. Sleepiness in the normal adolescent. In Sleep and Its Disorders in Children. Guilleminault, C. Ed.: 53-66. Raven Press. New York.

24. Blader, J.C., H.S. Koplewicz, H. Abikoff \& C. Foley. 1997. Sleep problems of elementary school children. Arch. Pediatr. Adolesc. Med. 151: 473-480.

25. Saarenpaa-Heikkila, O., P. Laippala \& M. Koivikko. 2000. Subjective daytime sleepiness in schoolchildren. Family Pract. 17: 129-133.

26. Carskadon, M.A.. 1982. The second decade. In Sleeping and Waking Disorders: Indications and Techniques. Guilleminault, C. Ed.: 99-125. Addison Wesley. Menlo Park.

27. Van Den Bulck, J. 2004. Television viewing, computer game playing, and Internet use and self-reported time to bed and time out of bed in secondary-school children [see comment]. Sleep 27: 101-104.

28. Owens, J., R Maxim, M. McGuinn, et al. 1999. Television-viewing habits and sleep disturbance in school children. Pediatrics 104: e27.

29. Van Den Bulck, J. 2007. Adolescent use of mobile phones for calling and for sending text messages after lights out: results from a prospective cohort study with a one-year follow-up. Sleep 30: 1220-1223.

30. Crowley, S.J., C. Acebo, M.A. Carskadon, et al. 2007. Sleep, circadian rhythms, and delayed phase in adolescence. Sleep Med. 8: 602-612.

31. Czeisler, C., G. Richardson, R. Coleman, et al. 1981. Chronotherapy: resetting the circadian clocks of patients with delayed sleep phase insomnia. Sleep 4: 1-21.

32. Guilleminault, C., C.C. McCann, M. Quera-Salva \& M. Cetel. 1993. Light therapy as treatment of dyschronosis in brain impaired children. Eur. J. Pediatr. 152: 754 759.

33. Okawa, M., M. Uchiyama, S. Ozaki, et al. 1998. Circadian rhythm sleep disorders in adolescents: clinical trials of combined treatments based on chronobiology. Psychiatry Clin. Neurosc. 52: 483-490.

34. Patois, E., J. Valatz \& A. Alperovitch. 1993. Prevalence of sleep and wakefulness disorders in high school students at the Academy of Lyon. Rev. Epiedmiol. Sante Publique 41: 383-388.

35. Ledoux, S., M. Choquet \& R. Manfredi. 1994. Selfreported use of drugs for sleep or distress among French adolescents. J. Adolesc. Health 15: 495-502.

36. Ivanenko, A., V.M. Crabtree, R. Tauman \& D. Gozal. 2003. Melatonin in children and adolescents with insomnia: a retrospective study. Clin. Pediatr. 42: 51-58.

37. Szeinberg, A., K. Borodkin, Y. Dagan, et al. 2006. Melatonin treatment in adolescents with delayed sleep phase syndrome. Clin. Pediatr. (Phila) 45: 809-818.

38. Smits, M., H. Van Stel, K. Van Der Heijen, et al. 2003. Melatonin improves health status and sleep in children with idiopathic chronic sleep-onset insomnia: a randomized, placebo-controlled trial. J. Am. Acad. Child Adolesc. Psychiatry 42: 1286-1293.

39. Stores, G. 2003. Medication for sleep-wake disorders. Arch. Dis. Child. 88: 899-903.

40. Hublin, C., J. Kaprio, M. Partinen \& M. Koskenvu. 2001. Parasomnias: co-occurrence and genetics. Psychiatr. Genet. 11: 65-70.

41. Lecendreux, M., C. Bassetti, Y. Dauvilliers, et al. 2003. HLA and genetic susceptibility to sleepwalking. Mol. Psychiatry 8: 114-117. 
42. Guilleminault, C., L. Palombini, R. Pelayo, et al. 2003. Sleepwalking and sleep terrors in prepubertal children: what triggers them? Pediatrics 111: e17-e25.

43. Laberge, L., R.E. Tremblay, F. Vitaro \& J. Montplaisir. 2000. Development of parasomnias from childhood to early adolescence. Pediatrics 106: 67-74.

44. Klackenberg, G. 1982. Somnambulism in childhoodprevalence, course and behavioral correlations. A prospective longitudinal study (6-16 years). Acta Paediatrica Scandinavica 71: 495-499.

45. Frank, N.C., A. Spirito, L. Stark \& J. Owens-Stively. 1997. The use of scheduled awakenings to eliminate childhood sleepwalking. J. Pediatr. Psychol. 22: 345353.

46. Durand, V.M. \& J.A. Mindell. 1999. Intervention for childhood sleep terrors. Behav. Ther. 30: 705-715.

47. Remulla, A., C. Guilleminault, A. Remulla \& C. Guilleminault. 2004. Somnambulism (sleepwalking). Expert Opin. Pharmacother. 5: 2069-2074.

48. Pesikoff, R. \& P. Davis. 1971. Treatment of pavor nocturnus and somnambulism in children. Am. J. Psychiatry 128: $778-781$.

49. Smedje, H., J.E. Broman, J. Hetta, et al. 1999. Parents' reports of disturbed sleep in 5-7-year-old Swedish children. Acta Paediatrica 88: 858-865.

50. Butler, R.J., P. Holland, S. Gasson, et al. 2007. Exploring potential mechanisms in alarm treatment for primary nocturnal enuresis. Scand. J. Urol. Nephrol. 41:407-413.

51. Lottmann, H.B. \& I. Alova. 2007. Primary monosymptomatic nocturnal enuresis in children and adolescents. Int. J. Clin. Pract. Supplement(155): 8-16.

52. Ferrara, P., G. Marrone, V. Emmanuele, et al. 2008. Homotoxicological remedies versus desmopressin versus placebo in the treatment of enuresis: a randomised, double-blind, controlled trial [see comment]. Pediatr. Nephrol. 23: 269-274.

53. Neveus, T., K. Tullus, T. Neveus \& K. Tullus. 2008. Tolterodine and imipramine in refractory enuresis; a placebo-controlled crossover study. Pediatr. Nephrol. 23: 263-267.

54. Larney, V. \& R. Dwyer. 2006. Hyponatraemic convulsions and fatal head injury secondary to desmopressin treatment for enuresis. Eur. J. Anaesthesiol. 23: 895897.

55. Stone, J., P.S. Malone, D. Atwill, et al. 2008. Symptoms of sleep-disordered breathing in children with nocturnal enuresis. J. Pediatr. Urol. 4: 197-202.

56. Weider, D.J. \& P.J. Hauri. 1985. Nocturnal enuresis in children with upper airway obstruction. Int. J. Pediatr. Otorhinolaryngol. 9: 173-182.
57. Weissbach, A., A. Leiberman, A. Tarasiuk, et al. 2006. Adenotonsilectomy improves enuresis in children with obstructive sleep apnea syndrome. Int. J. Pediatr. Otorhinolaryngol. 70: 1351-1356.

58. Rajaram, S.S., A.S. Walters, S.J. England, et al. 2004. Some children with growing pains may actually have restless legs syndrome. Sleep 27: 767-773.

59. Chervin, R.D., K.H. Archbold, J.E. Dillon, et al. 2002. Associations between symptoms of inattention, hyperactivity, restless legs, and periodic leg movements. Sleep 25: 213-218.

60. Picchietti, D., R.P. Allen, A.S. Walters, et al. 2007. Restless legs syndrome: prevalence and impact in children and adolescents-the Peds REST study [see comment]. Pediatrics 120: 253-266.

61. Cortese, S., E. Konofal, M. Lecendreux, et al. 2005. Restless legs syndrome and attention-deficit/hyperactivity disorder: a review of the literature. Sleep 28: 1007-1013.

62. Picchietti, D.L., D.J. Underwood, W.A. Farris, et al. 1999. Further studies on periodic limb movement disorder and restless legs syndrome in children with attention-deficit hyperactivity disorder. Mov. Disord. 14: 1000-1007.

63. Picchietti, D.L., S.J. England, A.S. Walters, et al. 1998. Periodic limb movement disorder and restless legs syndrome in children with attention-deficit hyperactivity disorder. J. Child Neurol. 13: 588-594.

64. Muhle, H., A. Neumann, K. Lohmann-Hedrich, et al. 2008. Childhood-onset restless legs syndrome: clinical and genetic features of 22 families. Mov. Disord. 23: 1113-1121; quiz 203.

65. Oner, P., E.B. Dirik, Y. Taner, et al. 2007. Association between low serum ferritin and restless legs syndrome in patients with attention deficit hyperactivity disorder. Tohoku J. Exp. Med. 213: 269-276.

66. Picchietti, M.A., D.L. Picchietti, M.A. Picchietti \& D.L. Picchietti. 2008. Restless legs syndrome and periodic limb movement disorder in children and adolescents. Semin. Pediatr. Neurol. 15: 91-99.

67. Kryger, M.H., K. Otake \& J. Foerster. 2002. Low body stores of iron and restless legs syndrome: a correctable cause of insomnia in adolescents and teenagers. Sleep Med. 3: 127-132.

68. Konofal, E., I. Arnulf, M. Lecendreux, et al. 2005. Ropinirole in a child with attention-deficit hyperactivity disorder and restless legs syndrome. Pediatr. Neurol. 32: 350-351.

69. Walters, A.S., D.E. Mandelbaum, D.S. Lewin, et al. 2000. Dopaminergic therapy in children with restless legs/periodic limb movements in sleep and ADHD. 
Dopaminergic Therapy Study Group. Pediatr. Neurol. 22: 182-186.

70. Hoban, T.F. 2003. Rhythmic movement disorder in children. Cns Spectrums 8: 135-138.

71. Klackenberg, G. 1971. Rhythmic movements in infancy and early childhood. Acta Paediatrica Scandinavica 224(suppl): 74-82.

72. Hoban, T.F. Sleep related rhythmic movement disorder. In The Parasomnias and Other Sleep-related Movement Disorders. Thorpy, M. \& G. Plazzi, Eds. Cambridge University Press. Cambridge. In Press.

73. Guilleminault, C., F.L. Eldridge, F.B. Simmons \& W.C. Dement. 1976. Sleep apnea in eight children. Pediatrics 58: $23-30$.

74. Iber, C., S. Ancoli-Israel, A. Chesson \& S.F. Quan. 2007. The AASM Manual for the Scoring of Sleep and Associated Events. American Academy of Sleep Medicine. Westchester.

75. Guilleminault, C., R. Pelayo, D. Leger, et al. 1996. Recognition of sleep-disordered breathing in children. Pediatrics 98: 871-882.

76. Marcus, C.L. 2001. Sleep-disordered breathing in children. Am. J. Respir. Crit. Care Med. 164: 16-30.

77. Shott, S.R., R. Amin, B. Chini, et al. 2006. Obstructive sleep apnea: Should all children with Down syndrome be tested? Arch. Otolaryngol. - Head Neck Surg. 132: 432-436.

78. Ng, D.K., H.N. Hui, C.H. Chan, et al. 2006. Obstructive sleep apnoea in children with Down syndrome. Singapore Med. J. 47: 774-779.

79. Gozal, D. 1998. Sleep-disordered breathing and school performance in children. Pediatrics 102: 616-620.

80. Chervin, R.D., D.L. Ruzicka, B.J. Giordani, et al. 2006. Sleep-disordered breathing, behavior, and cognition in children before and after adenotonsillectomy. Pediatrics 117: e769-e778.

81. Li, A.M., C.T. Au, R.Y. Sung, et al. 2008. Ambulatory blood pressure in children with obstructive sleep apnoea: a community based study. Thorax 63: 803-809.

82. Leung, L.C., D.K. Ng, M.W. Lau, et al. 2006. Twentyfour-hour ambulatory BP in snoring children with obstructive sleep apnea syndrome. Chest 130: 1009-1017.

83. Cox, M.A., G.L. Schiebler, W.J. Taylor, et al. 1965. Reversible pulmonary hypertension in a child with respiratory obstruction and cor pulmonale. J. Pediatr. 67: 192-197.

84. Sofer, S., E. Weinhouse, A. Tal, et al. 1988. Cor pulmonale due to adenoidal or tonsillar hypertrophy or both in children. Noninvasive diagnosis and follow-up. Chest 93: 119-122.
85. Marcus, C.L., S. Curtis, C.B. Koerner, et al. 1996. Evaluation of pulmonary function and polysomnography in obese children and adolescents. Pediatr. Pulmonol. 21: 176-183.

86. Kalra, M., T. Inge, V. Garcia, et al. 2005. Obstructive sleep apnea in extremely overweight adolescents undergoing bariatric surgery. Obes. Res. 13: 1175-1179.

87. Marcus, C.L. \& C.L. Marcus. 2002. Total energy expenditure in children with obstructive sleep apnoea syndrome [comment]. Eur. Respir. J. 19: 12151216.

88. Grigg-Damberger, M., D. Gozal, C.L. Marcus, et al. 2007. The visual scoring of sleep and arousal in infants and children. J. Clin. Sleep. Med. 3: 201-240.

89. Rosen, C.L., L. D’Andrea \& G.G. Haddad. 1992. Adult criteria for obstructive sleep apnea do not identify children with serious obstruction. Am. Rev. Respir. Dis. 146(5 Pt 1): 1231-1234.

90. Zaremba, E.K., M.E. Barkey, C. Mesa, et al. 2005. Making polysomnography more "child friendly:" a familycentered care approach. J. Clin. Sleep. Med. 1: 189-198.

91. Mangat, D., W.C. Orr \& R.O. Smith. 1977. Sleep apnea, hypersomnolence, and upper airway obstruction secondary to adenotonsillar enlargement. Arch. Otolaryngol. 103: 383-386.

92. Section on Pediatric Pulmonology SoOSASAAoP. 2002. Clinical practice guideline: diagnosis and management of childhood obstructive sleep apnea syndrome [see comment]. Pediatrics 109: 704-712.

93. Brietzke, S.E. \& D. Gallagher. 2006. The effectiveness of tonsillectomy and adenoidectomy in the treatment of pediatric obstructive sleep apnea/hypopnea syndrome: a meta-analysis. Otolaryngol. Head Neck Surg. 134: 979984.

94. Tauman, R., T.E. Gulliver, J. Krishna, et al. 2006. Persistence of obstructive sleep apnea syndrome in children after adenotonsillectomy. J. Pediatr. 149: 803-808.

95. Guilleminault, C., Y.S. Huang, C. Glamann, et al. 2007. Adenotonsillectomy and obstructive sleep apnea in children: a prospective survey. Otolaryngol. Head Neck Surg. 136: 169-175.

96. Guilleminault, C., M. Partinen, J.P. Praud, et al. 1989. Morphometric facial changes and obstructive sleep apnea in adolescents. J. Pediatr. 114: 997-999.

97. Amin, R., L. Anthony, V. Somers, et al. 2008. Growth velocity predicts recurrence of sleep-disordered breathing 1 year after adenotonsillectomy. Am. J. Respir. Crit. Care Med. 177: 654-659.

98. O’Donnell, A.R., C.L. Bjornson, S.G. Bohn \& V.G. Kirk. 2006. Compliance rates in children using noninvasive 
continuous positive airway pressure. Sleep 29: 651658.

99. Massa, F., S. Gonsalez, A. Laverty, et al. 2002. The use of nasal continuous positive airway pressure to treat obstructive sleep apnoea. Arch. Dis. Child. 87: 438443.

100. Kheirandish-Gozal, L., D. Gozal, L. Kheirandish-Gozal \& D. Gozal. 2008. Intranasal budesonide treatment for children with mild obstructive sleep apnea syndrome. Pediatrics 122: e149-e155.

101. Mansfield, L.E., G. Diaz, C.R. Posey \& J. Flores-Neder. 2004. Sleep disordered breathing and daytime quality of life in children with allergic rhinitis during treatment with intranasal budesonide. Ann. Allergy Asthma Immunol. 92: 240-244.

102. Kotagal, S. 1996. Narcolepsy in children. Semin. Pediatr. Neurol. 3: 36-43.

103. Smit, L.S., G.J. Lammers \& C.E. Catsman-Berrevoets. 2006. Cataplexy leading to the diagnosis of NiemannPick disease type C. Pediatr. Neurol. 35: 82-84.

104. Tobias, E.S., J.L. Tolmie \& J.B. Stephenson. 2002. Cataplexy in the Prader-Willi syndrome. Arch. Dis. Child. 87: 170 .
105. Vendrame, M., N. Havaligi, C. Matadeen-Ali, et al. 2008. Narcolepsy in children: a single-center clinical experience. Pediatr. Neurol. 38: 314-320.

106. Morgenthaler, T.I., V.K. Kapur, T. Brown, et al. 2007. Practice parameters for the treatment of narcolepsy and other hypersomnias of central origin. Sleep 30: 17051711.

107. Wise, M.S., D.L. Arand, R.R. Auger, et al. 2007. Treatment of narcolepsy and other hypersomnias of central origin. Sleep 30: 1712-1727.

108. Murali, H. \& S. Kotagal. 2006. Off-label treatment of severe childhood narcolepsy-cataplexy with sodium oxybate. Sleep 29: 1025-1029.

109. Arnulf, I., L. Lin, N. Gadoth, et al. 2008. Kleine-Levin syndrome: a systematic study of 108 patients. Ann. Neurol. 63: 482-493.

110. Arnulf, I., M. Lecendreux, P. Franco \& Y. Dauvilliers. 2008. [Kleine-Levin syndrome: state of the art]. Rev. Neurol. (Paris) 164: 658-668.

111. Portilla, P., E. Durand, A. Chalvon, et al. 2002. [SPECTidentified hypoperfusion of the left temporomesial structures in a Kleine-Levin syndrome]. Rev. Neurol. (Paris) 158(5 Pt 1): 593-595. 